\title{
Manufacturing of Polymer-based 1×2 Y-branch Symmetric and Asymmetric Waveguide Coupler through Moulding Technique
}

\author{
M.S.M. Ghazali1 ${ }^{1}$ F.R.M. Romlay ${ }^{{ }^{*}}$ and A.A. Ehsan ${ }^{2}$ \\ ${ }^{1}$ Machine Manufacturing Union in Mechatronics Laboratory, Manufacturing Focus Group, Faculty of Mechanical and Automotive Engineering \\ Technology, Universiti Malaysia Pahang, 26600 Pahang, Malaysia \\ 2Institute of Microengineering and Nanoelectronics (IMEN), Universiti Kebangsaan Malaysia,43600 Selangor, Malaysia
}

ABSTRACT - Manufacturing of Y-branch coupler depends on high technology production equipment and in-factory accuracy assembly tools. The manufacturing of a $1 \times 2$ Y-branch symmetric and asymmetric waveguide coupler based on the mould replication process and Epoxy OG142 as an optical core is presented; an alternative to provide a less complex technique. The polymer optical waveguide adopted two basic designs: the $1 \times 2$ Y-branch symmetric coupler as the core structure and the $1 \times 2$ asymmetric coupler that allows non-symmetric optical splitting. This paper focused on the main structure fabrication of the $1 \times 2$ symmetric and asymmetric waveguide coupler that produces a power output. The fabrication was done by engraving acrylic to produce a master mould using CNC machining tools for optical devices. Both $1 \times 2$ devices were made via soft lithography, which duplicated the pattern from the master mould onto a second mould to produce an actual device. Optical polymer epoxy OG142 was injected into the second mould, of which the product was then put on top of acrylic. The device was completed after curing the optical polymer glue, epoxy OG142, by exposing the assembly on the second mould under UV light until both parts bonded. The difference between the simulation and design TOFR value was only $\pm 2 \%$. This showed that the simulation and design are in good agreement, which provides similar performance.
ARTICLE HISTORY

Received: $6^{\text {th }}$ April 2021

Revised: $6^{\text {th }}$ May 2021

Accepted: $16^{\text {th }}$ Aug 2021

KEYWORDS

Waveguide;

Polymer optical;

Soft lithography

\section{INTRODUCTION}

Plastic optical fibres (POFs) for waveguides have been explored started in the 1960s [1]. POFs are often used as a medium for optical data communication. POFs offer the advantage of being practical to use and easy handling with robust characteristics. Various polymers specifications make it possible to design optical fibres and waveguides with higher performance capabilities. An advantage of using polymers as optical fibres is that the polymers offset intrinsic and extrinsic loss mechanisms, which cause upgraded specification than conventional glass fibres [2]. Polymer optical fibres nowadays are produced from interesting new materials, giving it an economic advantage particularly in entertainment, which heavily relies on light transmission and sensor systems [3]. POFs can be categorised into two parts which are active and passive components. Active components such as receivers and transmitters, while passive components are types of optical devices like filters, connectors, couplers, and splitters [4].

Couplers and splitters as passive devices, are of great interest in short length networks for applications in separating transmitted and received signals of telephone lines, in distributed systems for cable TV, and optical sensors for security systems. The application of combining or splitting optical signals in an optical device to complete its designated function is a commonly required feature. Coupler and splitter designs had been reported by [5], [6] and [7]. One type of splitter produced by these designers is the Y-branch splitter, which has great impact in applications like the splitting of propagated light. Previously, Y-branch splitters were normally fabricated by polishing a couple of fibres and attach both together. Other techniques to construct Y-branch splitters include hot embossing and microinjection techniques. The splitter application is also applied in multimode interconnects on the nanophotonics area [11]. However, all these techniques require high technology production equipment and in-factory precision assembly tools.

Many research works were executed via soft lithography, which is an alternative technique to fabricate the Y-branch splitter. The soft lithography technique can be defined as a manufacturing technique that makes patterns mechanically with a polydimethylsiloxane (PDMS) master stamp. The technique adopts a simple concept of pattern transfer using a master mould to deform or pattern the additive material. This paper reports the fabrication of a polymer-based $1 \times 2 \mathrm{Y}$ branch symmetric and asymmetric waveguide coupler as an optical splitter device. The master mould was designed by acrylic plastic, shaped through high-speed CNC machining. This master mould is then utilised to produce the $1 \times 2 \mathrm{Y}$ branch splitter according to the soft lithography technique. The acrylic plastic-based device is manufactured as the waveguide core by the optically clear polymer of epoxy-based Epo-Tek OG142. Fabrication of the $1 \times 2$ Y-branch splitter is elaborated as follows. 


\section{DEVICE FUNDAMENTAL AND SPECIFICATIONS}

The asymmetrical waveguide coupler design is a basis of a tap off ratio (TOFR) to divide light coming from the core bus line [5]. The concept of asymmetric Y-branch splitter that divided the incoming light can be set up in a multimode device [6]. Figure 1 shows the illustration of a $1 \times 2$ symmetric $Y$ junction splitter with the bus and tap line design by computational design. The splitting angle is set at $18^{\circ}$. Both the spatial and s-bend splitters obtained a good splitting performance [7]. From the design, it is ideal to produce various designs of asymmetric waveguide couplers with any different size of tap width.

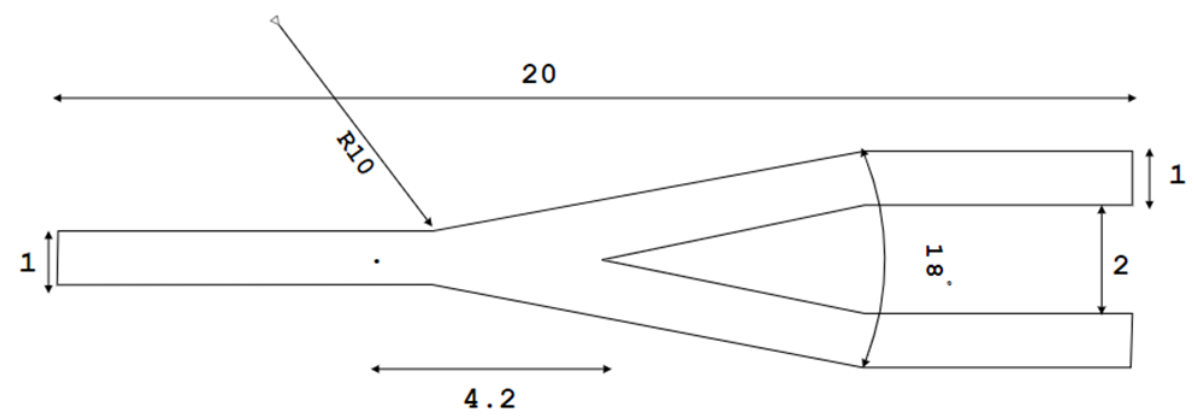

Figure 1. Y-branch symmetric waveguide coupler parameter design by CAD [10].

The symmetric Y-branch splitter with TOFR and linear taper is shown in Figure 2. The TOFR output power exiting at the end of the waveguide is provided by Eq. (1) [8].

$$
\text { TOFR }=\frac{y}{y+x}
$$

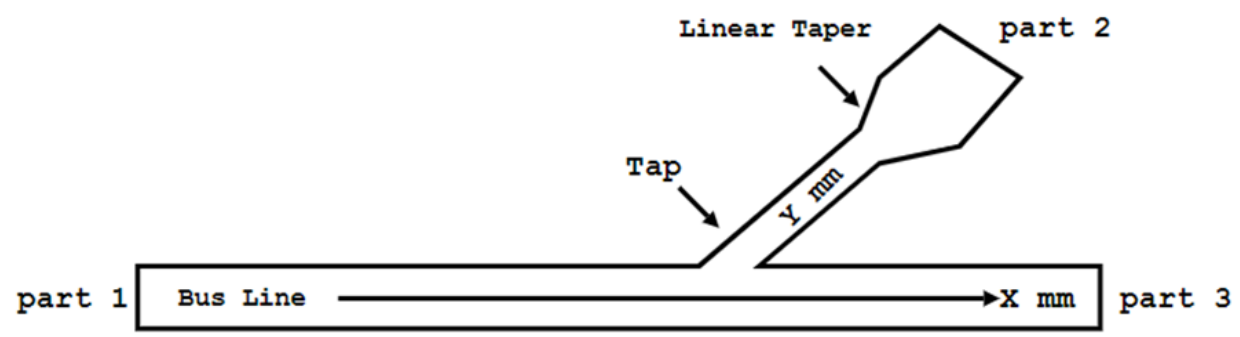

Figure 2. Asymmetric Y-branch waveguide coupler with tap and bus lines [10].

The $1 \times 2$ waveguide coupler design utilised a single branch as the main bus line at a fixed 1 mm of core width dimension. The second branch was the taper line at various width dimensions. By altering the taper width, many arrangements of the power output are possible to be produced. Figure 3 shows the $1 \times 2$ asymmetric polymer waveguide coupler together with the tap and bus line. From the design, three example different tap line sizes are created as shown in Figure 4 which are $50 \%, 40 \%$, and 30\% TOFR. The widths of the taper size are $1 \mathrm{~mm}, 0.65 \mathrm{~mm}$ and $0.45 \mathrm{~mm}$, respectively.

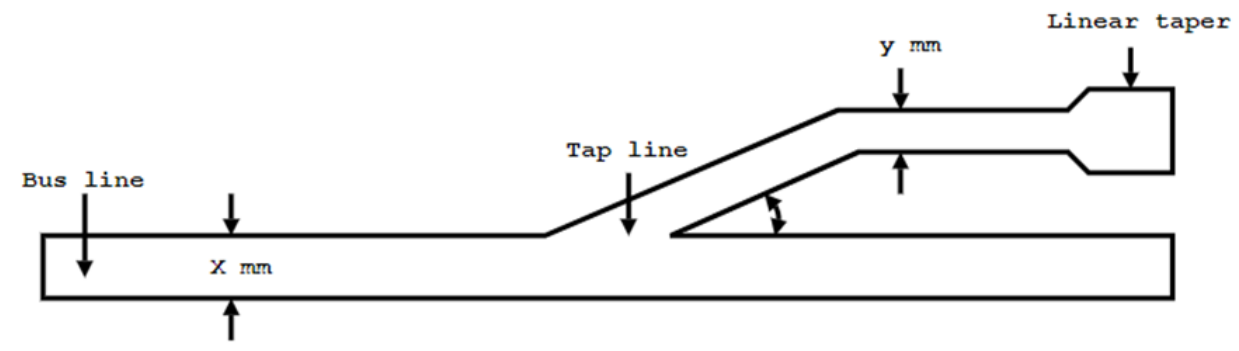

Figure 3. $1 \times 2$ asymmetric polymer waveguide coupler geometry property [10]. 


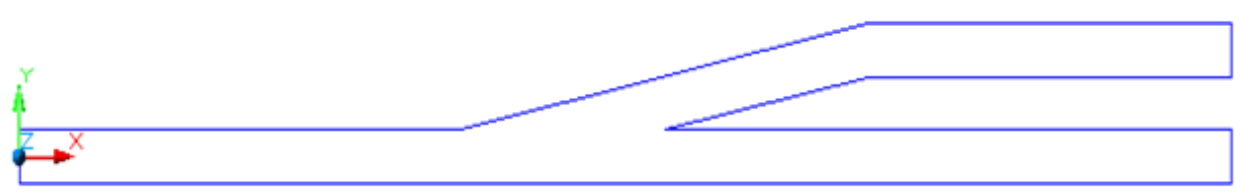

(a)

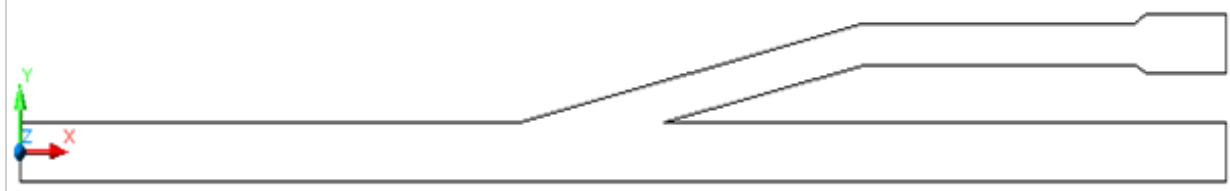

(b)

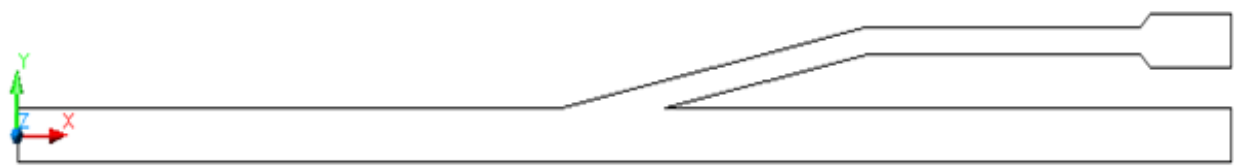

(c)

Figure 4. Waveguide coupler; (a) 50\% TOFR; (b) 40\%; (c) 30\%TOFR.

\section{Material and Methods}

Several chemicals and materials were applied throughout the fabrication of the $1 \times 2 \mathrm{Y}$-branch splitter, including PDMS, curing agent for the PDMS, Epo-Kwick, and optical polymer OG142 as the core for the splitter. The optical polymer OG142 perform a refractive index of 1.57 , a haze of $0.2 \%$ and light transmissions of $92 \%$. The material used as a substrate for the splitter was PMMA or acrylics. It has a refractive index of 1.49 , a haze of $0.5 \%$ and light transmissions of $92 \%$ [9].

\section{Fabrication Process}

The manufacture of the $1 \times 2$ Y-branch symmetric and asymmetric waveguide coupler consisted of two steps; using Epo-Kwick and without using Epo-Kwick. The reason for using Epo-Kwick was to obtain the sharp Y profile to reduce high loss during light propagation. Before using Epo-Kwick, the first step was to construct the polymer-based $1 \times 2 \mathrm{Y}$ branch splitter. A $5 \mathrm{~mm}$ thick acrylic was used as a substrate holder; meanwhile, the acrylic substrate was also cut into $20 \times 26 \mathrm{~mm}$ and $16 \times 20 \mathrm{~mm}$ rectangles. After cleaning the holder and substrate, a Roland EGX 400 Desktop CNC cutting machine was operated to produce the parts. The $0.5 \mathrm{~mm}$ and $1 \mathrm{~mm}$ diameters of the end-mill were used for the cutting with machine rotation of $30000 \mathrm{rpm}$. After engraving, the acrylic substrate was put into the substrate holder and proceeded with the preparation of the Epo-Kwick solution. The solution was prepared with a 5:1 mixture of resin to hardener, which is considered ideal for this method. Therefore, $10 \mathrm{ml}$ of hardener to $50 \mathrm{ml}$ of resin were mixed and stirred slowly until its colour turned crystal-like. The mixture was then applied to the holder until flushed.

The epoxy was allowed to harden for approximately 40-45 minutes before the sample was released from the holder. The sample was obtained by separating the acrylic master mould and the Epo-Kwick material; the Epo-Kwick material was then put back into the holder. This process was to produce the $\mathrm{v}$-shape of the $1 \times 2 \mathrm{Y}$-branch splitter because the smallest end-mill tool of the CNC machine could engrave only up to $0.5 \mathrm{~mm}$. Duplication of the pattern from the EpoKwick material was achieved utilising a PDMS stamp. The mixture ratio for the PDMS stamp was 10:1; 10 g of PDMS to $1 \mathrm{~g}$ of curing agent for the PDMS was mixed using an electronic weight scale. Afterwards, these two solutions were agitated for 10 minutes using a glass rod. The stirring introduced bubbles to the mixture. A cup with the PDMS was placed in a vacuum desiccator to remove these bubbles, which was evacuated for at least 30 minutes.

Once a clear, bubble-free PDMS mixture was obtained, the next step was to fill the PDMS mixture into the holder. A furnace was prepared by increasing the temperature to $70^{\circ} \mathrm{C}$. Then place the holder into the furnace and let it sit for 1 hour. Once the soft lithography process was over, the PDMS elastomer layer was carefully lifted from the holder and the Epo-Kwick material. Then, isopropanol (IPA) was used to soften the PDMS layer, thus easy for handling the layer to avoid any cracks of the PDMS elastomer. Upon releasing the patterned PDMS from the Epo-Kwick material, it was then put back into the holder. Optical polymer liquid Epo-Tek OG142 was slowly poured onto the PDMS pattern until it filled up the Y shape. The acrylic was placed on top of the PDMS pattern to acquire the $1 \times 2 \mathrm{Y}$-branch symmetric waveguide coupler, which was then cured under UV light with an intensity of $60 \mathrm{~W}$. Upon exposure to UV light, the optical polymer (OG142) would bond to the acrylic and produce a permanent sealing. Figure 5 presents the fabrication step of the $1 \times 2 \mathrm{Y}$ branch symmetric waveguide coupler. 


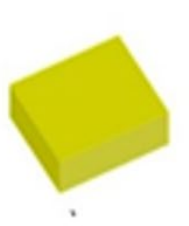

(a)

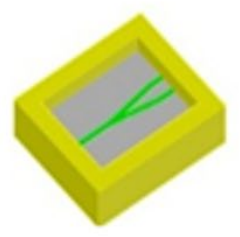

(1)

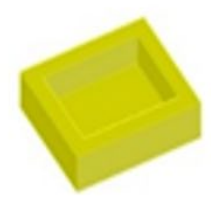

(b)

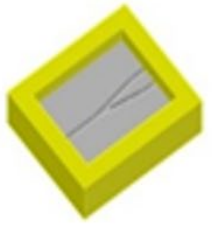

(k)

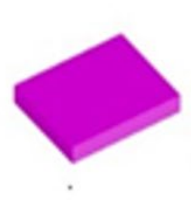

(c)

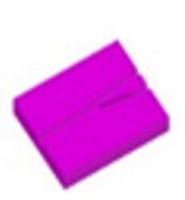

(d)

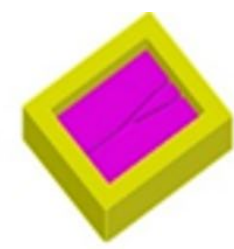

(e)

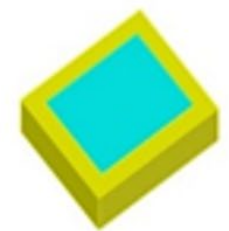

(f)

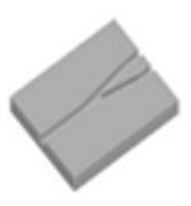

(j)

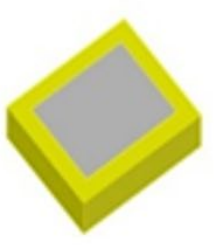

(i)

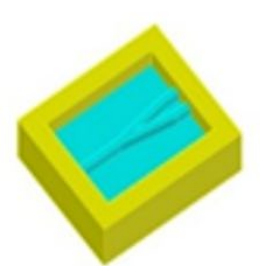

(h)

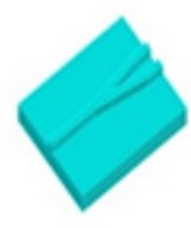

(g)

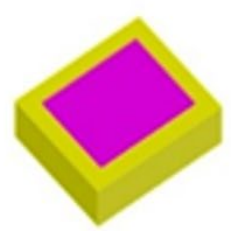

(m)

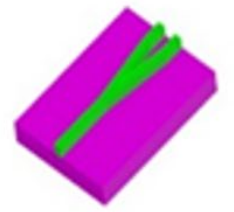

(n)

Figure 5. Fabrication steps of $1 \times 2$ Y-branch symmetric waveguide coupler utilising Epo-Kwick solution. (a) Cut and clean holder; (b) Engrave the holder; (c) Cut and clean the substrate; (d) Engrave the substrate; (e) Put the substrate into the holder; (f) Coat Epo-Kwick solution in the holder; (g) Pattern produced by Epo-Kwick solution; (h) Put the second pattern into the holder; (i) Coat the PDMS solution in the holder; (j) Pattern produced by PDMS solution; (k) Put the elastomer pattern into the holder; (1) Drop the optical polymer OG142 until it fills up the Y-shape; (m) Put the substrate

into the holder and expose under UV-light; (n) Final product of $1 \times 2$ Y-branch symmetric waveguide coupler

After completing the fabrication of a $1 \times 2$ Y-branch symmetric waveguide coupler with Epo-Kwick, the second step process was to fabricate the coupler without using Epo-Kwick. The procedures without using Epo-Kwick are similar to that of using Epo-Kwick but excluding any steps involving Epo-Kwick to produce the master mould and is illustrated in Figure 6. Upon completion of the soft lithography procedures, both $1 \times 2$ Y-branch symmetric waveguide devices were tested and analysed accordingly.

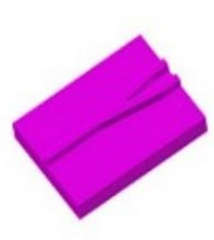

(a)

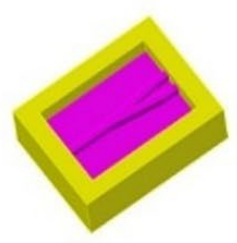

(b)

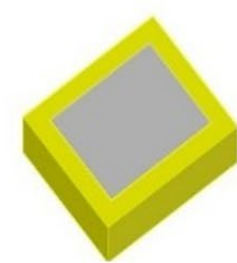

(c)

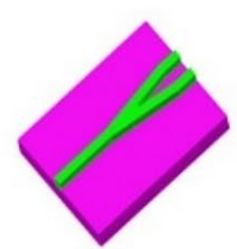

(h)

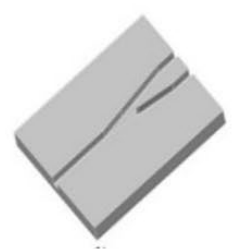

(d)

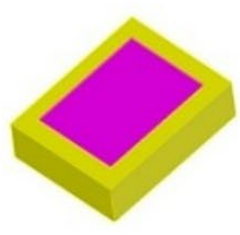

(g)

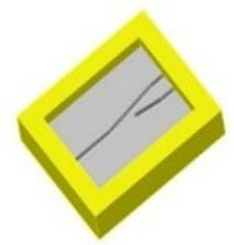

(e)

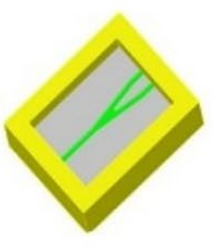

(f)

Figure 6. Fabrication flow of $1 \times 2$ Y-branch symmetric waveguide coupler without using Epo-Kwick solution. (a) Engrave the Y-shape pattern as a master mould; (b) Put the master mould into the holder; (c) Coat the holder with PDMS solution; (d) Pattern produced by the PDMS solution; (e) Put the PDMS elastomer into the holder; (f) Drop the optical polymer OG142 into the Y-shape; (g) Put the substrate into the holder and expose under UV-light; (h) Final device of $1 \times 2$ Y-branch symmetric waveguide coupler

\section{RESULTS AND DISCUSSION}

The simulation and fabrication of the $1 \times 2$ Y-branch symmetric and asymmetric waveguide couplers with several methods were discussed. The difference between both devices can be seen from the V-shape of the $1 \times 2 \mathrm{Y}$-branch 
symmetric waveguide couplers. Before ray-tracing was conducted, some simulation basis assumptions had to be determined. The simulation of the $1 \times 2$ Y-branch coupler was performed by applying the non-sequential ray-tracing technique. The optical source was a rectangular light with a wavelength of $650 \mathrm{~nm}$ and input power of $1.0 \mathrm{~mW}$. For ray tracing, the waveguide core was the optical polymer Epo-Tek OG142 with an index of refraction of 1.58.

The cladding for the waveguides was PMMA or acrylic with a 1.49 reflection index. Figure 7 presents the actual final device of the $1 \times 2 \mathrm{Y}$-branch symmetric waveguide coupler with the proposed core and cladding materials. It shows that the ray-tracing test was conducted on the assembled coupler which the two-dimensional (2D) ray tracing diagrams of the $1 \times 2$ Y-branch are shown in Figure 8(a) and 8(b). Figure 8(a) diagram that is not applied in Epo-Kwick mould shows higher losses than the application of Epo-Kwick mould, which indicated lower losses as in Figure 9.

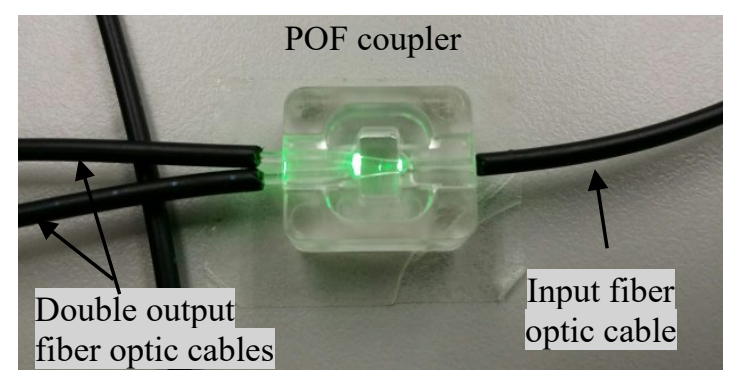

Figure 7. The fabricated devices assembly for the test.

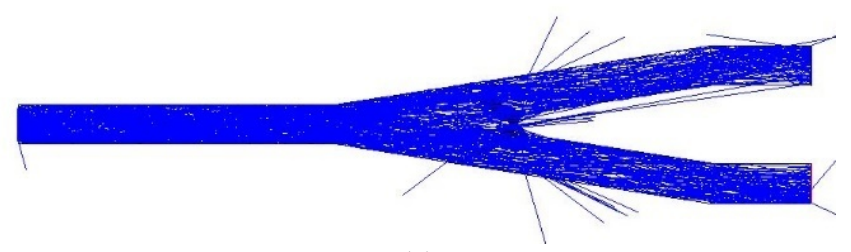

(a)

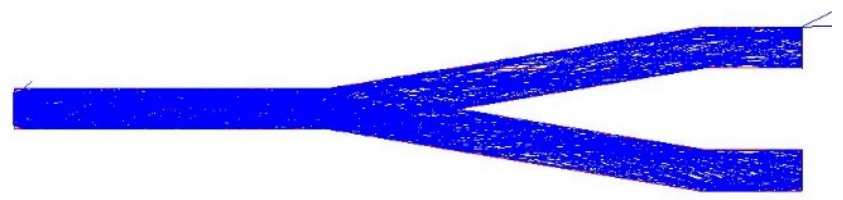

(b)

Figure 8. 2D ray tracing for $1 \times 2$ Y-branch symmetric waveguide coupler of (a) device 1 and (b) device 2 .

The output signals for both devices obtained at output ports 1 and 2 were 0.402 and $0.390 \mathrm{~mW}$ for device 1 , while output ports 1 and 2 were 0.489 and 0.487 for device 2 . The detector image for both devices at output ports 1 and 2 are shown in Figure 9.
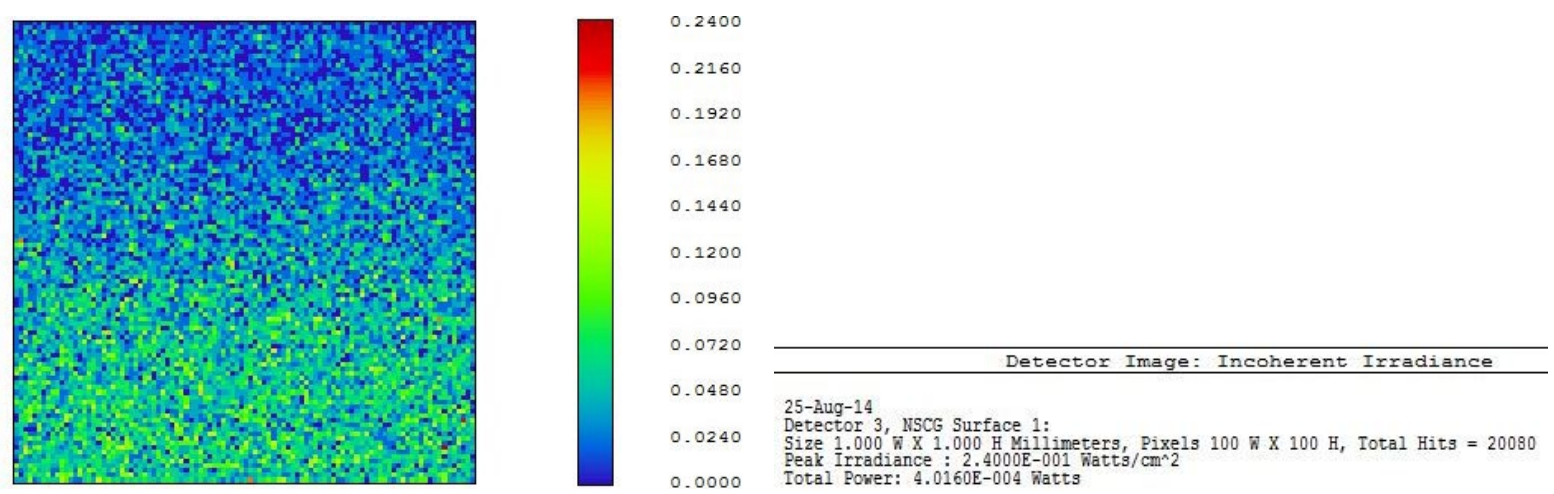

(a) 

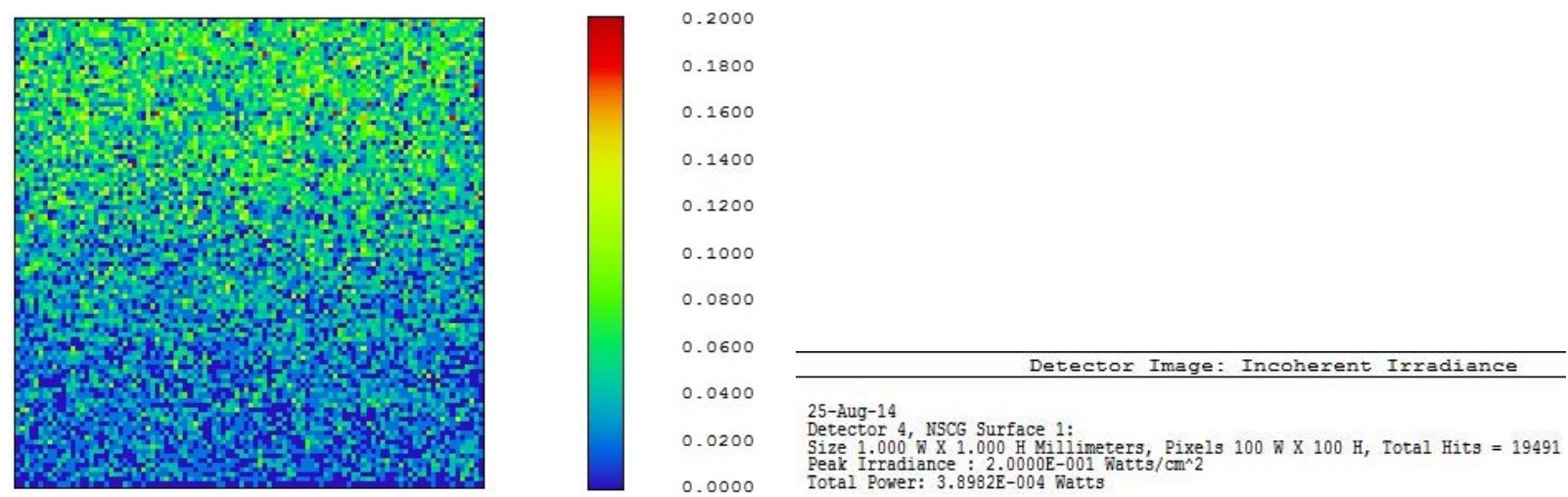

(b)
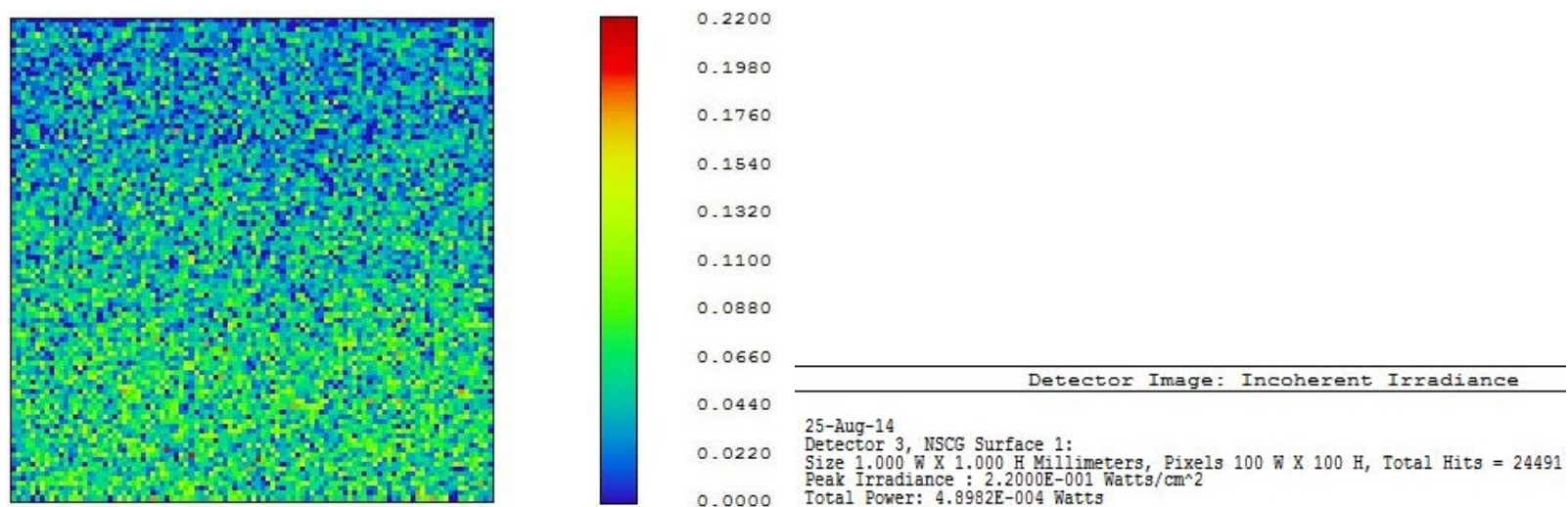

(c)
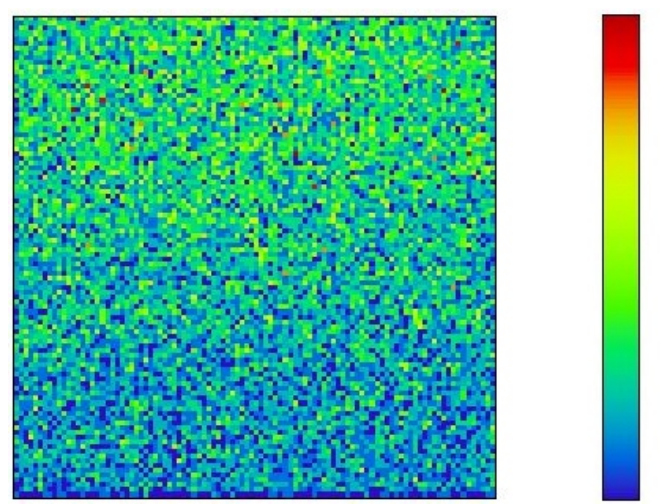

0.2200
0.1980
0.1760
0.1540
0.1320
0.1100
0.0880
0.0660
0.0440
0.0220
0.0000

$0.0000(d)$

Figure 9. Detector image for the $1 \times 2$ Y-branch asymmetric waveguide coupler (a) output port 1, device 1, (b) output port 2, device 1, (c) output port 1, device 2 and (d) output port 2, device 2 .

Based on device outputs from port 1 and port 2, the insertion loss for device 1 was about $4.1 \mathrm{~dB}$, whereas the insertion loss for device 2 was about $3.0 \mathrm{~dB}$. The excess loss of device 1 was about $1.0 \mathrm{~dB}$, and in device 2, approximately $0.1 \mathrm{~dB}$. The loss was determined based on the following equation [10]:

$$
\alpha=-10 \log \left(\left(P_{1}+P_{2}\right) / P_{\text {in }}\right)
$$

where, $P_{1}$ and $P_{2}$ are the power at the two outputs while $P_{\text {in }}$ is the output power.

The results showed that both devices could indeed perform as a $1.0 \mathrm{~dB}$ splitter. The value of the symmetric output ratio for section $\mathrm{Y}$ - splitter is $50 \%$. If the input power was $100 \%$, then the output power is $50 \%$ of the input. The fabricated $1 \times 2$ Y-branch asymmetric waveguide coupler in this research may serve as an alternative to those devices developed by Takezawa et al. [5], Mizuno et al. [6], and Kotzbuecher et al. [7]. These devices, as aforementioned, require expensive production equipment and have time-consuming procedural steps. The proposed fabrication technique that incorporates the soft lithography technique can produce the master mould, PDMS, optical polymer and UV-curable polymer for the waveguide. These results showed that both techniques were capable of providing approximately similar outputs with optical polymer, Epo-Tek OG142.

For the asymmetric waveguide coupler study, fifteen designs of the polymer waveguide coupler with different width tap sizes were reported. The software checked the ray tracing for optical performance and the code generation of the 
waveguide design to be forecasted. Applying the 3D design of the polymer waveguide coupler, the non-sequential ray tracing was done when the source light was applied to the input port that travelled through the waveguide. The wavelength used in the simulation was $650 \mathrm{~nm}$, with $1.0 \mathrm{~mW}$ of input power. When the light entered the input port of the waveguide, it reflected through the polymer of the waveguide. For the output port, the photodetectors set up in the software was functioned to detect the values of the output light intensities at both the branch of the waveguide, which were bus and tap lines. Three of the designs output ratios of the polymer waveguide coupler are shown as in Figure 10. The figure shows $31 \%, 44 \%$ and $50 \%$ of split waveguide output and the rest is produced by the 'bus' line. The reason for various percentages obtained was because of the different taper width sizes.

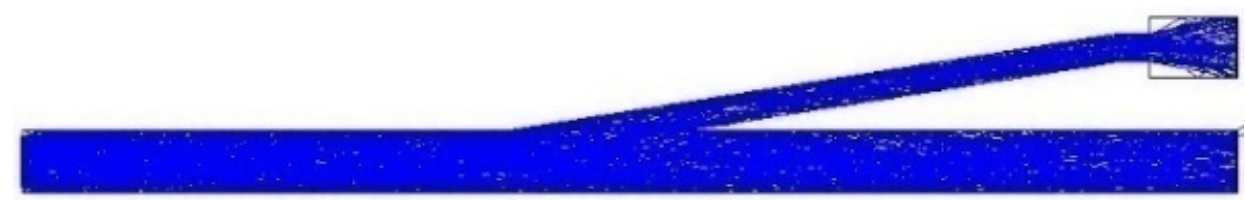

(a) $31 \%$

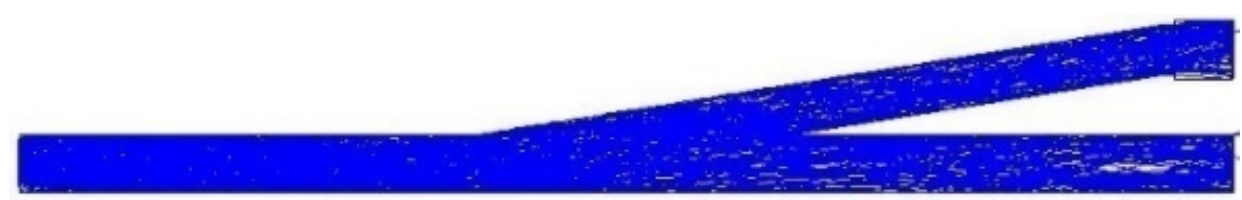

(b) $44 \%$

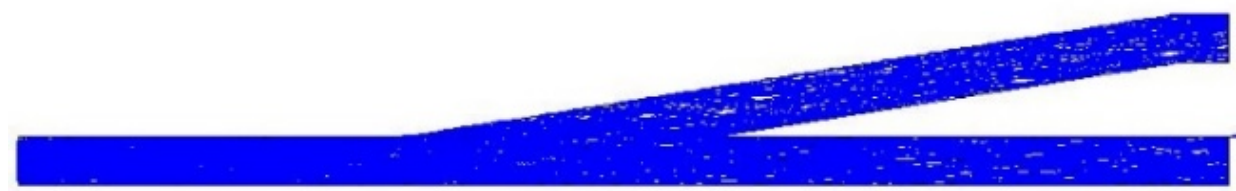

(c) $50 \%$

Figure 10. The $1 \times 2$ asymmetric waveguide coupler with (a) $450 \mu \mathrm{m}$ tap width obtained $31 \%$ TOFR (b) $650 \mu \mathrm{m}$ tap width obtained 44\% TOFR and (c) $1000 \mu \mathrm{m}$ tap width obtained 50\% TOFR.

The result shows that the higher tap width indicated a higher TOFR percentage. The reason is more light propagates at the higher cross-section area. This mean, tap width size is proportional to the TOFR percentage. The other tap width results of the $1 \times 2$ asymmetric waveguide coupler are given in Table 1 . The tap widths had to be set from $300 \mu \mathrm{m}$ up to 1 mm range for TOFR of $23 \%$ until $50 \%$.

Table 1. TOFR design and simulation comparison.

\begin{tabular}{cccc}
\hline \multirow{2}{*}{ No. } & \multirow{2}{*}{ Tap width $(\mu \mathrm{m})$} & \multicolumn{2}{c}{ TOFR } \\
\cline { 3 - 4 } & & Design $(\%)$ & Simulation $(\%)$ \\
\hline 1 & 300 & 23.1 & 21.1 \\
2 & 350 & 25.9 & 22.4 \\
3 & 400 & 28.6 & 28.2 \\
4 & 450 & 31.0 & 31.3 \\
5 & 500 & 33.3 & 34.7 \\
6 & 550 & 35.5 & 35.2 \\
7 & 600 & 37.5 & 38.4 \\
8 & 650 & 39.4 & 41.1 \\
9 & 700 & 41.2 & 42.3 \\
10 & 750 & 42.9 & 43.2 \\
11 & 800 & 44.4 & 45.4 \\
12 & 850 & 45.9 & 45.6 \\
13 & 900 & 47.4 & 45.3 \\
14 & 950 & 48.7 & 47.6 \\
15 & 1000 & 50.0 & 49.2 \\
\hline
\end{tabular}

Figure 11 shows the profile of the TOFR against the tap width for both the calculation design and simulation. The results of the calculated design TOFR were based on Eq. (1), from a range of $y$ from $300 \mu \mathrm{m}$ to $1000 \mu \mathrm{m}$ with a fixed bus line width, $x$ of $1000 \mu \mathrm{m}$. The simulation results were based on ray-tracing tools. 


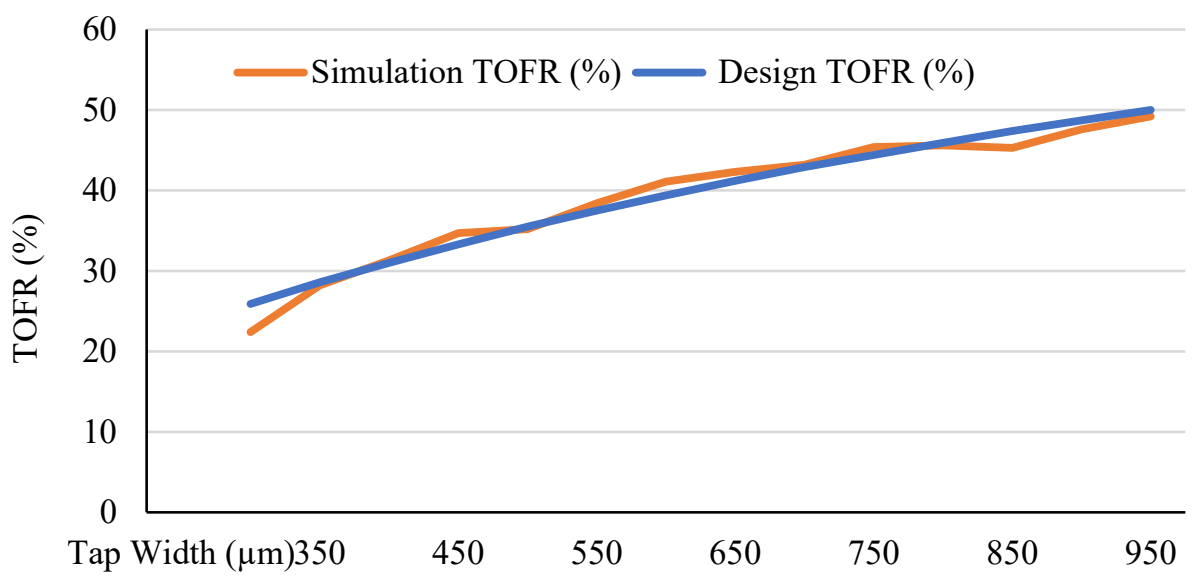

Figure 11. TOFR vs tap width for $1 \times 2$ asymmetric coupler (design and simulation).

The designed and simulation values of the TOFR showed that the error was minimal. The difference between the simulation and design TOFR value was only $\pm 2 \%$. This showed that the simulation and design are in good agreement, which provides similar performance. The simulated waveguide coupler performs a quite similar linear characteristic as per the calculated design. The simulation results of the TOFR for the $1 \times 2$ asymmetric waveguide coupler were ranged between $21.1 \%$ and $49.2 \%$. Thus, the developed model of the $1 \times 2$ POF coupler was considered acceptable for further application.

\section{CONCLUSION}

Fabrication and simulation of polymer-based $1 \times 2$ Y-branch symmetric and asymmetric waveguide couplers as splitters were presented. The $1 \times 2$ Y-branch splitters were designed and fabricated using optical polymer, OG142. Both devices gave an excellent performance as a $1 \mathrm{~dB}$ splitter. Moreover, the proposed device is able to be fabricated with accessible production techniques and high device throughput. A single master mould would even allow for the fabrication of the device multiple times. However, the limitation of the process comes from the higher optical polymer viscosity causing the bubble trap inside the cure waveguide. Some attention is required to handle the free-bubble construction of the device.

\section{ACKNOWLEDGEMENT}

This research was partially supported by research, development and commercialisation grant RDU172206 and UIC180302 of University Malaysia Pahang.

\section{REFERENCES}

[1] R. Tricker, Optoelectronic and Fiber Optic Technology. Oxford, U.K: Elsevier Science, 2002.

[2] I. Ayesta et al., "Fabrication of active polymer optical fibers by solution doping and their characterisation," Polymers, vol. 11, pp. 52, 2019, doi:10.3390/polym11010052.

[3] M. Hernaez et al., "Optical fibre sensors using graphene-based materials: A review," Sensors, vol. 17, pp. 155, 2017, doi: $10.3390 / \mathrm{s} 17010155$

[4] O. Zieman et al., POF Handbook. Berlin: Springer, 2008.

[5] C. Burtscher, D Seyringer, and A Kuzma,"Modeling and optimisation of $1 \times 32$ Y-branch splitter for optical transmission systems," Opt. Quantum Electron., vol. 49, pp. 396, 2017, doi: 10.1007/s11082-017-1228-8.

[6] H. Mizuno et al., "Compact y-branch-type polymeric optical waveguide devices with large-core connectable to plastic optical fibers,” Jpn. J. Appl. Phys., vol. 44, pp. 8504, 2005, doi: 10.1143/JJAP.44.8504.

[7] T. Klotzbuecher et al., "Fabrication of optical 1×2 POF couplers using the laser-LIGA technique," In Proc. SPIE, 2003, pp. 121.

[8] M. Loch, "Plastic optical fibers: properties and practical applications in optical transmission systems and equipment for WDM networking III," In Proc. SPIE, 2004, pp. 299-308.

[9] A.A. Ehsan et al., "Hollow optical waveguide coupler for portable access card system application," J. Opt. Commun, vol. 30, pp. 67-77, 2009.

[10] W.M. Henry and J.D. Love, “Asymmetric multimode Y-junction splitters,” Opt. Quantum Electron, vol. 29, pp. 379-392, 1997, doi: 10.1023/A:1018582912154.

[11] H. Xu, D. Dai, and Y. Shi, "Silicon integrated nanophotonic devices for on-chip multi-mode interconnects," Appl. Sci., vol. 10, no. 18 , pp. 10, 2020, doi: 10.3390/app10186365. 